\title{
Role of TRPV4 Channel in Human White Adipocytes Metabolic Activity
}

\author{
Julio C. Sánchez, Aníbal Valencia-Vásquez, Andrés M. García \\ Faculty of Health Sciences, Technological University of Pereira, La Julita, Pereira, Colombia
}

Background: Intracellular calcium $\left(\mathrm{Ca}^{2+}\right)$ homeostasis plays an essential role in adipocyte metabolism and its alteration is associated with obesity and related disorders. Transient receptor potential vanilloid 4 (TRPV4) channels are an important $\mathrm{Ca}^{2+}$ pathway in adipocytes and their activity is regulated by metabolic mediators such as insulin. In this study, we evaluated the role of TRPV4 channels in metabolic activity and adipokine secretion in human white adipocytes.

Methods: Human white adipocytes were freshly cultured and the effects of the activation and inhibition of TRPV4 channels on lipolysis, glucose uptake, lactate production, and leptin and adiponectin secretion were evaluated.

Results: Under basal and isoproterenol-stimulated conditions, TRPV4 activation by GSK1016709A decreased lipolysis whereas HC067047, an antagonist, increased lipolysis. The activation of TRPV4 resulted in increased glucose uptake and lactate production under both basal conditions and insulin-stimulated conditions; in contrast HC067047 decreased both parameters. Leptin production was increased, and adiponectin production was diminished by TRPV4 activation and its inhibition had the opposite effect.

Conclusion: Our results suggested that TRPV4 channels are metabolic mediators involved in proadipogenic processes and glucose metabolism in adipocyte biology. TRPV4 channels could be a potential pharmacological target to treat metabolic disorders.

Keywords: Adipocytes; TRPV4 protein, human; Metabolism; Lactates; Glucose

\section{INTRODUCTION}

Intracellular calcium signaling plays a critical role in adipocyte biology. For instance, calcium-sensing receptor activation leads to lipolysis inhibition by increasing the intracellular calcium concentration $\left(\left[\mathrm{Ca}^{2+}\right]_{i}\right)$ and regulating major lipolysis enzymes [1]. Additionally, intracellular calcium signaling is involved in leptin [2] and adiponectin secretion [3]. Furthermore, increased $\left[\mathrm{Ca}^{2+}\right]_{i}$ alters the differentiation of mesenchymal stem cells and preadipocytes to mature adipocytes [4]. In human adipocytes, the release of fatty acid-binding protein 4 , a cytosolic protein involved in fatty acid metabolism, is regulated by $\left[\mathrm{Ca}^{2+}\right]_{\mathrm{i}}[5]$.
Adipokines and insulin are known for their effects on energy homeostasis and cell survival. Leptin activates Janus kinase 3 (JAK3)/signal transducer and activator of transcription 2 (STAT2) and phosphoinositide 3-kinase (PI3K)/Akt/mechanistic target of rapamycin (mTOR) pathways, which are involved in cell survival and metabolism regulation [6]. The absence of insulin promotes production of proinflammatory cytokines such as tumor necrosis factor- $\alpha$ and interleukin 6 and preadipocyte differentiation [7]; furthermore, adiponectin improves glucose metabolism and reduces lipolysis, inflammation, and insulin resistance in 3T3-L1 adipocytes [8]. Adipokines regulate adipose triglyceride lipase (ATGL) and hormone-sensitive lipase (HSL),
Received: 23 June 2021, Revised: 14 August 2021, Accepted: 19 August 2021

Corresponding author: Julio C. Sánchez

Faculty of Health Sciences, Technological University of Pereira, AA 97, La Julita, Pereira 660003, Colombia

Tel: +57-6-3137300, Fax: +57-6-3213206, E-mail: jcsanchez@utp.edu.co
Copyright $\odot 2021$ Korean Endocrine Society

This is an Open Access article distributed under the terms of the Creative Commons Attribution Non-Commercial License (https://creativecommons.org/ licenses/by-nc/4.0/) which permits unrestricted non-commercial use, distribution, and reproduction in any medium, provided the original work is properly cited. 
which are essential enzymes in lipolysis and are regulated by AMP-activated protein kinase (AMPK) [9]. Adiponectin receptor 1 activation causes lipolysis inhibition by reducing the protein levels of protein kinase A (PKA) [10], suppressing PKAmediated HSL activation, and promoting inhibition of HSL by AMPK [11].

Transient receptor potential vanilloid 4 (TRPV4) channels are essential for intracellular signaling due to their high permeability to calcium and capacity to be activated by multiple stimuli [12]. Furthermore, TRPV4 participates in calcium-dependent responses in survival [13], proliferation [14] and differentiation [15]; particularly in harmful conditions associated with drug toxicity [16], ischemia [13], or proinflammatory environments [17]. TRPV4 is considered ubiquitous [18], and its expression and functioning have been demonstrated in brown and white adipocytes [19,20]. Knockout models of TRPV4 channels show increased oxidative metabolism and decreased adipocyte size [21]. Additionally, TRPV4 gene polymorphisms are associated with body mass index and the distribution of corporal fat mass [22]. The evidence of the TRPV4 effect on the upregulation of proinflammatory pathways could be explained by a dependent relationship with adipokines and insulin, which is reinforced by the fact that insulin inhibits TRPV4 in white adipocytes [19].

These facts highlight the need to better understand the relationship between adipokines and TRPV4 activity because leptin and resistin have known effects of promoting insulin resistance and the proliferation of white adipocytes unleashing the prometabolic disease pathway [23,24]. This study provides evidence of the effects of TRPV4 on metabolic activity and leptin and adiponectin production in human white adipocytes.

\section{METHODS}

\section{Patients}

The study was carried out following the Code of Ethics of the World Medical Association for experiments involving humans and in accordance with the Ethical Principles for Medical Research Involving Human Subjects outlined in the Declaration of Helsinki. The study was approved by the Bioethics Committee of the Universidad Tecnológica de Pereira (IRB: Acta 06/2012). Samples of subcutaneous adipose tissue were obtained from healthy nonobese subjects (ages between 30 and 50 years old) during planned surgery for reasons different from the study, such as elective plastic surgery, excluding liposuction, or hernia repairs. None of the patients had any metabolic disease. No selection was made for sex. All participants gave written informed consent before sample extraction.

\section{Chemicals and reagents}

All chemicals and solutions were obtained from Sigma-Aldrich (St. Louis, MO, USA), unless otherwise stated.

\section{Isolation and culture of preadipocytes}

Preadipocytes were isolated and cultured using a previous protocol with some modifications [25]. We obtained 10 to $20 \mathrm{~g}$ of adipose tissue from each patient. Immediately after extraction, this tissue was maintained at $4^{\circ} \mathrm{C}$ and preserved in $0.9 \%$ saline solution ( $\mathrm{pH} 7.4$ ) until the specimen arrived at the laboratory, where it was processed within 6 hours. Under sterile conditions, the tissue was cut into small pieces. The resulting fragments were incubated in Dulbecco's modified Eagle medium (DMEM) supplemented with glutamine (2 $\mathrm{mM})$, antibiotics (100 IU/mL penicillin, $100 \mathrm{mg} / \mathrm{mL}$ streptomycin, $200 \mathrm{mg} / \mathrm{mL}$ neomycin) and collagenase type $\mathrm{I}(2,000 \mathrm{U} / \mathrm{mL})$ for 90 minutes at $37^{\circ} \mathrm{C}$. At the end of this period, the solution was filtered and centrifuged at 5,000 rpm for 5 minutes. The supernatant was discarded, and the pellets were resuspended in erythrocyte lysis solution (155 mM NH4Cl, $20 \mathrm{mM}$ Tris-HCl, pH 7.2) for $5 \mathrm{~min}$ utes at $37^{\circ} \mathrm{C}$, and then centrifuged again. Then, the pellets were resuspended in culture medium 1 , which consisted of fresh DMEM supplemented with glutamine $(2 \mathrm{mM})$, antibiotics (100 IU/mL penicillin, $100 \mathrm{mg} / \mathrm{mL}$ streptomycin, and $200 \mathrm{mg} / \mathrm{mL}$ neomycin), nonessential amino acids (1\%), and fetal calf serum (FCS, 10\%). After dispersion by agitation, the cells were transferred to culture plates and plated at a density of $1 \times 10^{6}$ cells/ $\mathrm{mL}$ and incubated at $37^{\circ} \mathrm{C}$ and $5 \% \mathrm{CO}_{2}$. After 3 days, the cells were resuspended in DMEM without FCS and supplemented with glutamine, antibiotics, and nonessential amino acids as before. Following the addition of transferrin $(10 \mathrm{mg} / \mathrm{mL})$, hydrocortisone (100 $\mathrm{nM})$, triiodothyronine (1.3 nM), and insulin (500 $\mathrm{mU} / \mathrm{mL}$ ) as trophic factors, this solution was referred to as culture medium 2. The medium was changed every 3 to 4 days. After 6 to 10 medium changes (over 18 to 30 days), the oil droplets became visible in the cells. The cells were evaluated when these droplets reached a substantial size and after the cells had acquired their typical spherical form. At this point, the cells were resuspended in a fresh medium and counted. Subsequently, the cells were resuspended in culture medium 2 without insulin at a density of $1 \times 10^{6}$ cells $/ \mathrm{mL}$, before being used in experiments. Oil Red $\mathrm{O}$ staining assays were performed to monitor the characteristics of lipid droplets in these cultures. 


\section{Leptin and adiponectin measurements}

The leptin and adiponectin levels were quantified in supernatants employing commercial kits (KAP2381 DIAsource Leptin-EASIA kit, KAPME09 Adiponectin-EASIA kit, Louvain-la-Neuve, Belgium) through an enzyme-linked immunosorbent assay technique. The cells were centrifuged at 2,000 $\times \mathrm{g}$ for 10 minutes to remove debris and the supernatants were collected and stored at $-20^{\circ} \mathrm{C}$. Each assay was performed in duplicate, and the data obtained were treated as medians and interquartile ranges.

\section{Determination of lipolysis}

Glycerol measurements were used as an index of lipolysis in primary culture adipocytes under basal conditions and under stimulation with isoproterenol (IPT). For stimulated lipolysis, adipocytes were preincubated in DMEM, containing $2 \%$ fatty acid-free bovine serum albumin and $1 \mu \mathrm{M}$ IPT for 120 minutes at $37^{\circ} \mathrm{C}, 5 \% \mathrm{CO}_{2}$, and $95 \% \mathrm{O}_{2}$ in a humidified atmosphere. Then, the medium was replaced by the same fresh medium without IPT and incubated for 60 minutes. Glycerol released into the medium was measured by colourimetric absorbance at $540 \mathrm{~nm}$ with Free Glycerol Reagent and normalized to protein content, which was determined using the Pierce BCA assay kit (Thermo Scientific, Waltham, MA, USA). All experiments were performed in triplicates.

\section{Glucose uptake}

${ }^{14} \mathrm{C}$-U-D-glucose uptake was assessed in adipocytes, as described previously [26]. Briefly, adipocytes were preincubated for 15 minutes in culture medium 2 without glucose in the absence or presence of GSK1016709A (GSK, $100 \mathrm{nM}$ ), a TRPV4 agonist, or HC067047 (HC, $50 \mathrm{nM}$ ), a TRPV4 antagonist, and thereafter stimulated or not with insulin $(1,000 \mathrm{IU} / \mathrm{mL})$. Then, cells were treated with $0.86 \mathrm{mmol} / \mathrm{L}{ }^{14} \mathrm{C}$-U-D-glucose (PerkinElmer, Boston, MA, USA) for 15 minutes at $37^{\circ} \mathrm{C}$. After 45 minutes, glucose uptake was stopped by washing the cells twice with ice-cold PBS. The cell fraction was collected, dissolved in scintillation fluid (Optima Gold, PerkinElmer) and subjected to scintillation counting, employing a liquid scintillation analyser Tri-Carb 4910 TR (PerkinElmer). Glucose uptake was determined by the rate of transmembrane glucose transport and calculated using the following equation: cellular clearance of medium glucose $=$ (c.p.m. cells $\times$ volume $) /($ c.p.m. medium $\times$ cell number $\times$ time). Glucose uptake was normalized to protein content, which was determined using the Pierce BCA assay kit (Thermo Scientific). All experiments were performed in triplicates.

\section{Lactate secretion}

The lactate concentration was determined enzymatically using the hydrazine sink method as described previously [27]. Briefly, $10 \mu \mathrm{L}$ of medium sample was diluted in $90 \mu \mathrm{L}$ of water and then incubated with $100 \mu \mathrm{L}$ of assay buffer (1 M glycine, $0.4 \mathrm{M}$ hydrazine sulfate in $1 \mathrm{M} \mathrm{NaOH}$, and $2.5 \mathrm{~mm}$ ethylenediaminetetraacetic acid [EDTA], adjusted to $\mathrm{pH} 9.2$ with $\mathrm{NaOH}$ before the addition of $4 \mathrm{~mm} \mathrm{NAD}{ }^{+}$and 2 units $/ \mathrm{mL}$ lactate dehydrogenase). Reactions were light protected and incubated at room temperature for 1 hour, after which the absorbance of NADH was measured at $340 \mathrm{~nm}$. Medium samples without lactate dehydrogenase were included as a negative control. Lactate levels were quantified using a standard curve of lactate. Media lactate levels were normalised to protein content, which was determined using the Pierce BCA assay kit (Thermo Scientific). All experiments were performed in triplicates.

\section{Statistical analysis}

The data analysis was performed using the analysis tools available in GraphPad Prism 8.0 (GraphPad, La Jolla, CA, USA). The percent change was calculated for each experimental condition. The results are shown as the mean and standard error of the mean. Data were treated with unpaired Student's $t$ test analysis for comparisons between groups and one-way analysis of variance (ANOVA) with Holms-Sidak post hoc analysis was used for multiple group comparisons or the respective non-parametric test. Spearman's test was performed to establish correlations between glucose uptake and lactate production adjusted by experimental group. A correlation matrix was constructed with the variables assessed to identify metabolic correlations. All statistical tests were performed with two-tailed tests, and a $P<0.05$ was considered significant.

\section{RESULTS}

\section{Role of TRPV4 in basal and stimulated lipolysis}

Fig. 1A shows the measurements of lipolysis under basal conditions and Fig. 1B shows the measurements of lipolysis under IPT-stimulated conditions. The lipolysis rate was significantly decreased under basal and stimulated conditions by the effect of TRPV4 activation, similar to the adipogenic insulin effect; in contrast, lipolysis was significantly increased by the inhibition of TRPV4 under basal conditions but not under stimulation with IPT. 

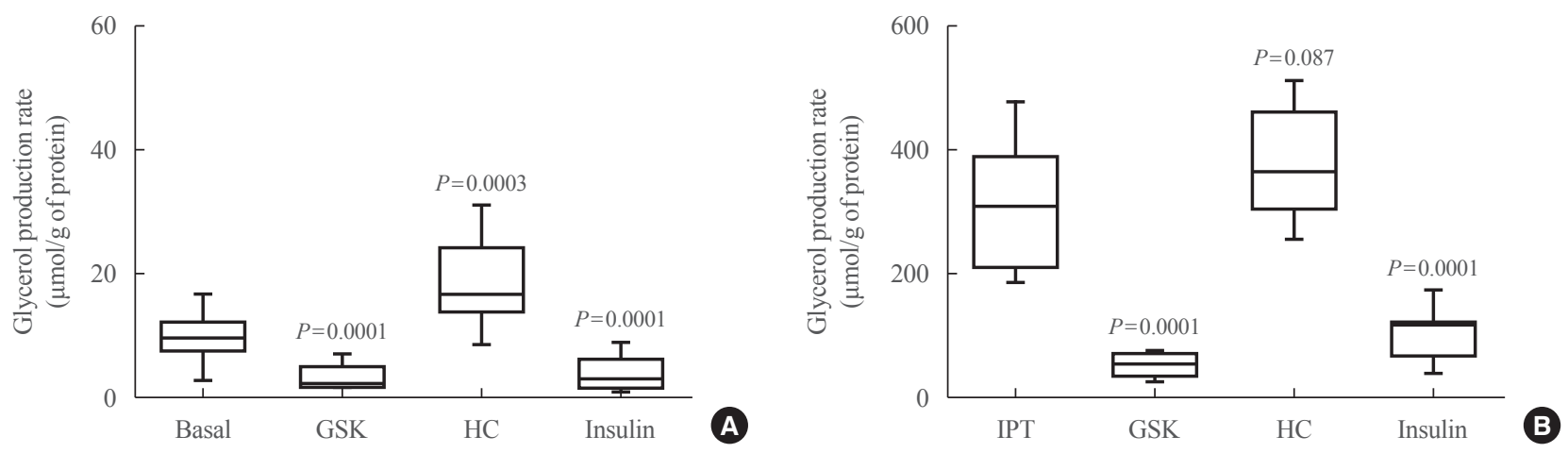

Fig. 1. Effect of transient receptor potential vanilloid 4 (TRPV4) effect on lipolysis. (A) Panel shows the effects of TRPV4 activation and inhibition on the glycerol production rate under basal conditions. (B) Panel shows the effect of TRPV4 on the glycerol production rate in isoproterenol (IPT)-stimulated conditions. The production rate was normalized to protein content. The data are expressed as median and interquartile range. All $P$ values are shown as an exact Mann-Whitney $U$ test value between TRPV4 activation and inhibition versus the basal and IPT-stimulated lipolysis rate ( $n=9$ in all cases). GSK, GSK1016709A; HC, HC067047.
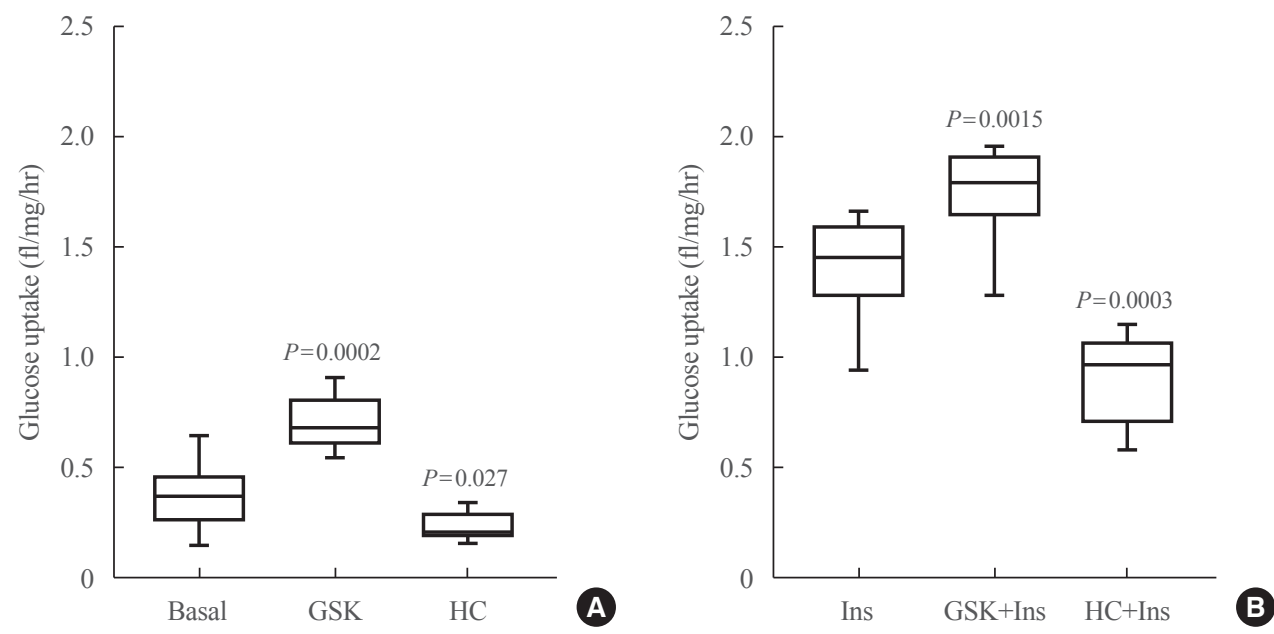

Fig. 2. Effect of transient receptor potential vanilloid 4 (TRPV4) on glucose uptake. (A) Panel shows the effects of TRPV4 activation and inhibition on glucose uptake under basal conditions. (B) Panel shows the effect of TRPV4 on glucose uptake under stimulation with insulin. The glucose uptake was normalized to protein content. The data are expressed as median and interquartile range. All $P$ values are shown as an exact Mann-Whitney $U$ test value between TRPV4 activation and inhibition versus basal and insulin-stimulated glucose uptake $(n=9$ in all cases). GSK, GSK1016709A; HC, HC067047; Ins, insulin.

\section{Effect of TRPV4 modulation on glucose uptake and lactate production}

Figs. 2A, 3A show the measurements of glucose uptake and lactate under basal conditions and Figs. 2B, 3B show the measurement under insulin exposure. The activation of TRPV4 in both scenarios significantly increased the glucose uptake and lactate production, conversely, the inhibition of TRPV4 decreased this uptake and production.

\section{Glucose uptake and lactate production as a linear relationship and the role of TRPV4 on this interaction}

A Spearman's test was performed in an XY plot constructed with glucose uptake as $\mathrm{X}$-axis variable and lactate production as Y-axis variable. A positive correlation was identified in all experimental groups $(r>0.90, P<0.0001)$. With these results, a linear regression model was designed to assess the effect of TRPV4 modulation on the correlation of glucose uptake vs. lactate production.

Fig. 4 shows the linear plots obtained under all the experimental conditions tested and explained in the Methods section. The respective slope decreased under the effect of insulin without any other agent present, which means that the effect of this hormone is not the same on glucose uptake and lactate production, being predominant on glucose uptake. Furthermore, al- 

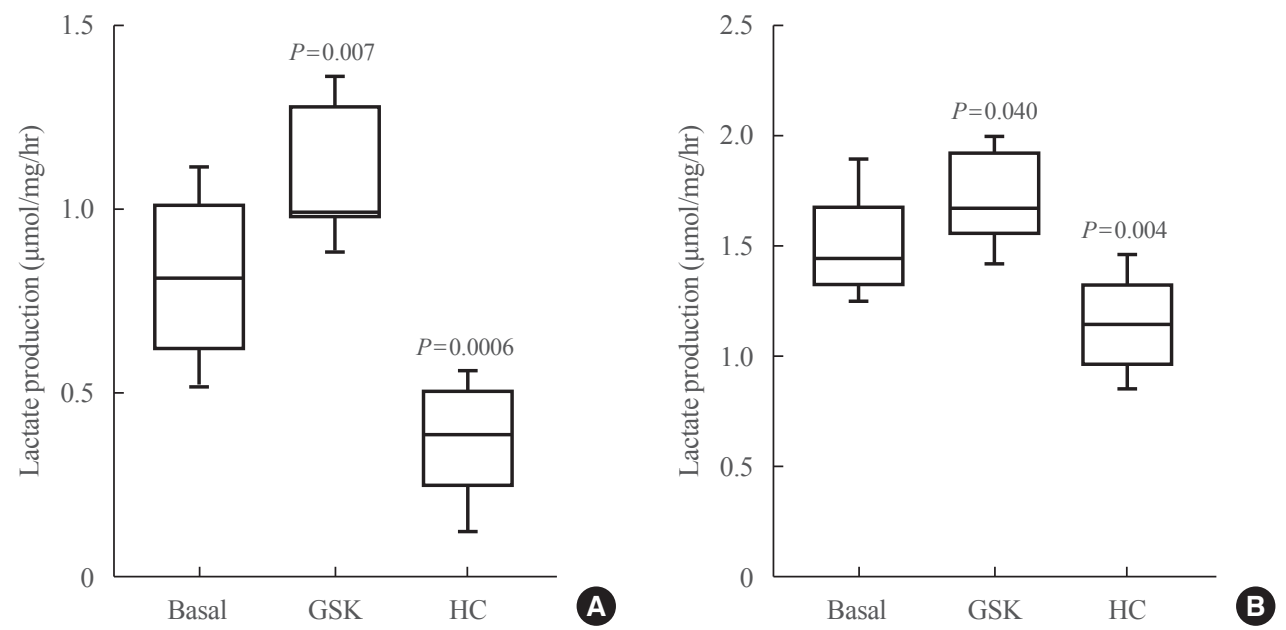

Fig. 3. Transient receptor potential vanilloid 4 (TRPV4) effect on lactate production. (A) Panel shows the effect of TRPV4 activation and inhibition on lactate secretion in basal conditions. (B) Panel shows the effect of TRPV4 on lactate secretion under stimulation with insulin. The lactate secretion was normalized to protein content. The data are expressed in median and interquartile range. All $P$ values are shown as an exact Mann-Whitney $U$ test value between TRPV4 activation and inhibition versus the basal and insulin-stimulated lactate production ( $n=9$ in all cases). GSK, GSK1016709A; HC, HC067047.

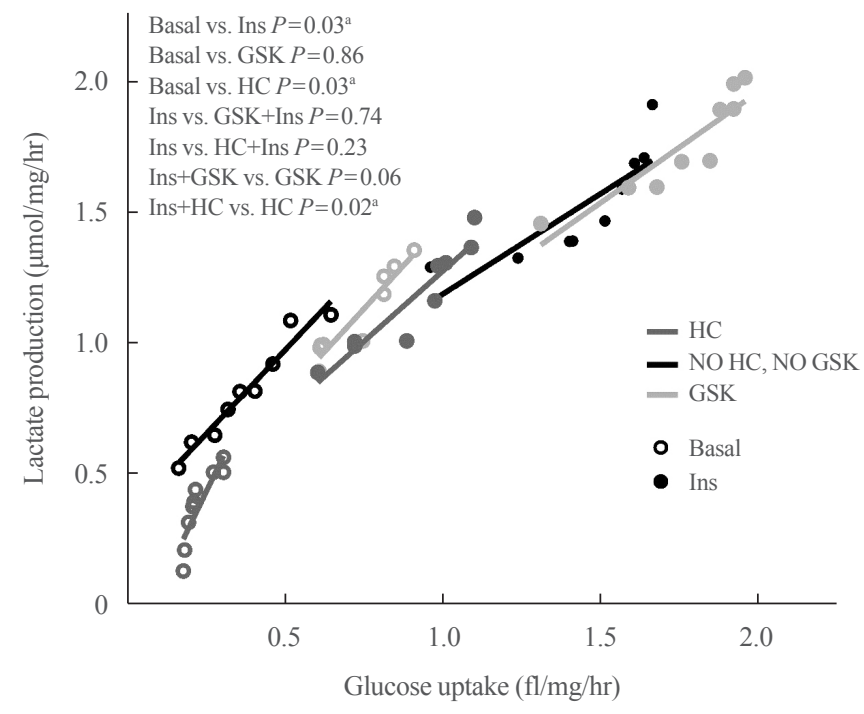

Fig. 4. The effect of transient receptor potential vanilloid 4 (TRPV4) on the linear relationship between glucose uptake and lactate production in all the experimental conditions, as indicated. The individual values are presented as separate points ( $n=9$ in all cases). Ins, insulin; GSK, GSK1016709A; HC, HC067047. 'Denotes significance.

though GSK shifts the linear plots to an upper right position (which reflects that this agonist increases both glucose uptake and lactate production) and $\mathrm{HC}$ shifts the linear plots to a lower left position (which reflects that this antagonist decreases both glucose uptake and lactate production), the effect of GSK and $\mathrm{HC}$ on the slopes in both basal and insulin-stimulated conditions are different since GSK causes the deletion of the insulin-in- duced difference in the slopes, which suggest that GSK has a predominant effect on lactate production. In contrast, HC increased the slope in basal conditions promoting lactate production over glucose uptake, but the effect is equilibrated by insulin. These findings suggest that TRPV4 channels play a dual role in these metabolic parameters, which are conditioned by insulin.

\section{Role of TRPV4 in leptin and adiponectin production}

The production of leptin was increased by the activation of TRPV4, congruently the inhibition of TRPV4 decreased leptin levels (Fig. 5A). For the adiponectin measurements, the activation of TRPV4 decreased this hormone levels and its inhibition produced the opposite effect (Fig. 5B).

\section{DISCUSSION}

The present study contributes to what is known regarding the role of TRPV4 channels in lipolysis, glucose uptake, lactate secretion, leptin secretion, and adiponectin secretion in human white adipocytes.

$\left[\mathrm{Ca}^{2+}\right]_{\mathrm{i}}$ plays important roles in fundamental processes for adipocytes, such as lipolysis [28]. TRPV4 channels are highly expressed in adipose tissue [29], and they are involved in $\left[\mathrm{Ca}^{2+}\right]_{\mathrm{i}}$ homeostasis in adipocytes [30]. Furthermore, we previously reported the functional expression of TRPV4 and the relevance of this channel as a significant $\mathrm{Ca}^{2+}$ pathway, which is regulated by 

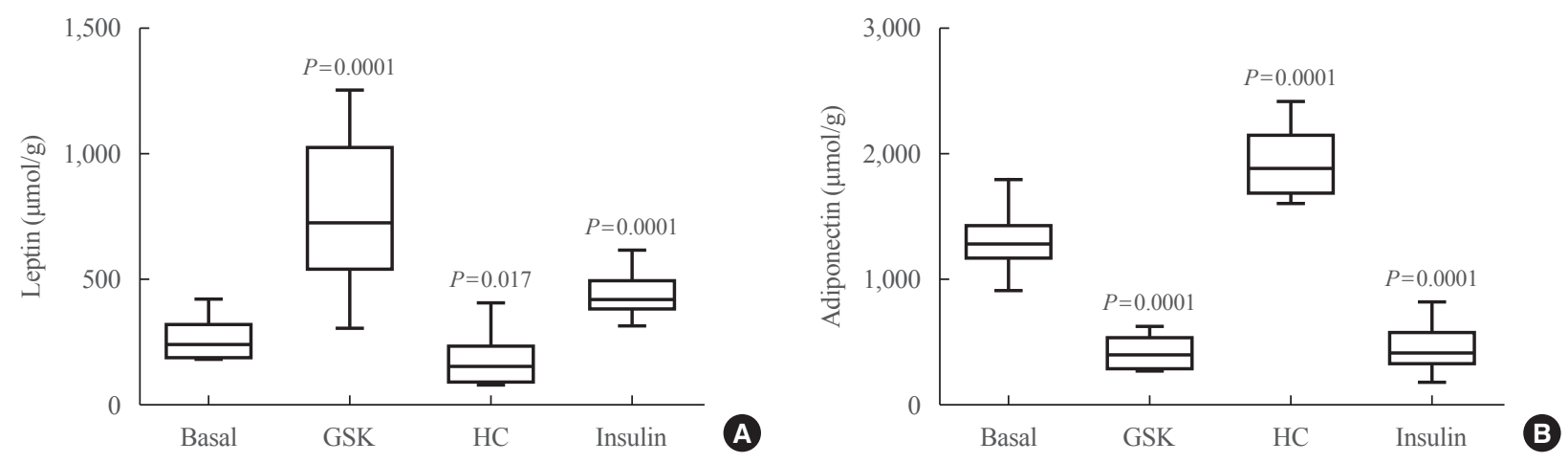

Fig. 5. Effect of transient receptor potential vanilloid 4 (TRPV4) and insulin on hormone production. (A) Panel shows the effects of TRPV4 activation and inhibition on leptin production under basal conditions. (B) Panel shows the effect of TRPV4 on adiponectin production. Hormone production was normalized to protein content. The data are expressed as median and interquartile range. All $P$ values are shown as an exact Mann-Whitney $U$ test between TRPV4 activation and inhibition versus basal hormone production ( $n=9$ in all cases). GSK, GSK1016709A; HC, HC067047.

insulin, in human white adipocytes [19]. Those findings suggest that TRPV4 channels are metabolic mediators of adipocyte biology, which is confirmed here.

Previously, it was demonstrated that TRPV4 inhibition in bone marrow adipocytes increases ATGL and HSL expression levels and produces subsequent lipolysis increase during basal and IPT-stimulated conditions [31], similar to our results. In white adipocytes, TRPV4 channel activation reduced the expression of peroxisome proliferator-activated receptor $\gamma$ coactivator $1 \alpha$ (PGC-1 $\alpha)$ [20], a key transcription factor that regulates lipolysis via ATGL and HSL expression [9]. A $\left[\mathrm{Ca}^{2+}\right]_{\mathrm{i}}$ increase inhibits lipolysis through phosphodiesterase 3B (PDE3B) activation, diminishes cyclic adenosine monophosphate (cAMP) levels, and suppresses PKA-induced HSL activation [28,32], which promote the inhibition of HSL by AMPK [11]. We hypothesized that the inhibitory role of TRPV4 channels on lipolysis could be associated with that effect on PDE3B, following the TRPV4-mediated $\left[\mathrm{Ca}^{2+}\right]_{\mathrm{i}}$ increase, which may lead to the activation of extracellular signal-regulated kinase 1/2 (ERK1/2) [20], and then to PCG- $1 \alpha$ suppression and the subsequent reduction of ATGL and HSL expression; however, this hypothesis needs to be further explored.

Glucose is a promoter of metabolic activity in adipocytes since insulin requires sufficient levels of glucose to promote lipogenesis [33], and induce a cascade of early phosphorylation of enzymes related to glycolysis in addition to the effect on GLUT4 translocation, which suggests that lipid metabolism mediated by insulin is a glucose-dependent metabolic process [34]. Additionally, adipocyte lactate production is a glucose-independent metabolic function, even during an insulin resistance scenario, but is also a parameter for the efficiency of the glycolytic pathway that drives the activation of gluconeogenic metabolism routes [27]. Insulin increases both glucose uptake and lactate production in adipocytes; lactate has an identified interaction as a signaling molecule through binding with an orphan G protein-coupled receptor (GPR81) [35], which has a known insulin-dependent antilipolytic effect. The activation of GPR81 by lactate induces the inhibition of adenylyl cyclase and subsequent cAMP formation, producing positive feedback given the activation of PDE3B by the PI3K/Akt signaling pathway of insulin [36].

We found that TRPV4 activation generates an insulin-like effect, including increased glucose uptake and lactate production, suggesting that TRPV4 channels are mediators of anabolic processes during basal conditions. For instance, the depletion of $\left[\mathrm{Ca}^{2+}\right]_{\mathrm{i}}$ alters glucose uptake in 3T3-L1 adipocytes [37]. $\mathrm{Ca}^{2+}-$ binding proteins could amplify this $\mathrm{Ca}^{2+}$ signaling. Interestingly, calcineurin inhibitors reduce glucose uptake through an increased GLUT4 internalization rate under basal and insulinstimulated conditions in human adipocytes [38,39], and their activation promotes lipolysis [40]. GLUT4 vesicle trafficking is mediated via the ERK1/2 signaling pathway [41]. Furthermore, TRPV1, another member of the TRPV channel family, has been associated with glucose uptake in adipocytes $[42,43]$. These intracellular effectors could be associated with TRPV4 channels to regulate glucose metabolism in human adipocytes; however, this hypothesis needs to be further evaluated.

It is important to note that the effects of the activation and inhibition of TRPV4 were differential between glucose uptake and lactate production, which was evidenced by the analysis of 
the linear plots under the different experimental conditions. These findings suggest that TRPV4 has an independent effect on lactate production, which is not proportional to the effect on glucose uptake. Lactate production do not exclusively depend on glucose availability or glucose metabolism [27]; TRPV4 could have a role in the regulation of this glucose-independent mechanism, although the pathways involved remain elusive.

Insulin has a negative regulatory effect on TRPV4 channel activity in white adipocytes [19]. However, our results showed that TRPV4 activation and insulin produce a synergistic effect on glucose uptake and lactate production. These metabolic effects could be explained by the fact that insulin did not completely abolish TRPV4 currents; some $\mathrm{Ca}^{2+}$ influx can activate PDE3B, previously activated by insulin signaling, leading to a further increase in glucose uptake followed by more lactate production. On the other hand, to some extent, this represents an unknown metabotropic activity in a synergic interaction with insulin receptor or GPR81 by lactate signaling. This hypothesis requires further studies, including assessments of the signaling pathway expression of both receptors.

Additionally, we identified a new regulatory role for TRPV4 channels in leptin secretion in human white adipocytes. TRPV4 activation produced an increase in leptin production, which is the opposite effect of the specific antagonist. These findings agree with previous results from mouse adipocytes, where TRPV4 knockdown resulted in reduced leptin expression [20]. Given that an increase in $\left[\mathrm{Ca}^{2+}\right]_{i}$ secondary to $\mathrm{Ca}^{2+}$ influx is required for stimulated leptin secretion [44] and TRPV4 activation is sufficient to increase $\left[\mathrm{Ca}^{2+}\right]_{\mathrm{i}}$ in white adipocytes [19], the mechanism of the stimulatory effect of the TRPV4 agonist on leptin secretion demonstrated here, can be explained.

Conversely, adiponectin secretion was diminished by TRPV4 activation and insulin stimulation. Similar findings were reported from adipocytes with a silenced TRPV4 gene, where ERK1/2 activation is recruited for PGC-1 $\alpha$ inhibition [20]. Interestingly, TRPC1/5 channels are regulators of adiponectin secretion: its activation and the subsequent increase in $\left[\mathrm{Ca}^{2+}\right]_{\mathrm{i}}$ inhibit adiponectin secretion [3]. Previous studies have demonstrated a functional interaction between TRPV4 and TRPC1 channels, a heteromeric complex that can produce $\mathrm{Ca}^{2+}$ influx when activated [45]. This interaction could take place to regulate adiponectin secretion.

3T3-L1 adipocytes with TRPV4 deletion presented vastly diminished ERK1/2 activation and leptin expression [20]. Furthermore, the inhibition of ERK1/2 decreases the insulin-stimulated leptin secretion in rat epididymal adipocytes [46], and in- hibitors of MAPK kinase (MERK), an upstream kinase of ERK1/2 signaling, reversed the reduced expression and blood levels of adiponectin [47]. Additionally, adiponectin expression is present in adipocytes deficient in TRPV4 and is associated with a reduction in ERK1/2 activity [20]. Given that ERK1/2 mediates signal transduction from TRPV4 channels and is associated with leptin and adiponectin production, MAPK signaling seems to be a common intracellular pathway in which TRPV4 channels and adipocyte biology take place.

These findings enhance the evidence of the role of TRPV4 as a proinflammatory and adipogenic mediator that may participate in the mechanisms of energy expenditure imbalance, especially in the context of stress and proinflammatory stimuli that can activate TRPV4 and promote leptin secretion and adipocyte anabolism. Furthermore, the increased leptin secretion by TRPV4 activation could explain the orexigenic disturbances and changes in behavioral satiety patterns in an insulin-independent mechanism [20,22]. The TRPV4 knockout mice models showed increased resistance to obesity and insulin sensitivity [21]; the polymodal profile of TRPV4 activation could be a route for explaining how environmental, inflammatory, behavioral, and stress-related conditions induce insulin resistance without necessarily high-glucose/high-fat diet conditioning.

The elucidation of the interactions of insulin and TRPV4 could expand the understanding of mechanisms for energy homeostasis and the consequences of hyperglycemia and insulin resistance, as well as the argument for proposing TRPV4 pharmacological modulation in different tissues to prevent organ damage or symptomatic control in the context of insulin resistance [48]. From middle-term perspective, TRPV4 pharmacological antagonism should be explored as a potential therapy for the treatment of obesity due to its role in lipolysis and leptin production, as well as in acute hyperglycemic disturbance scenarios such as diabetic ketoacidosis, in the context of insulin-induced glycolytic mechanism failure, as TRPV4 modulation could prevent hyperlactatemia and restore energy homeostasis through an insulin-like effect, especially as clinical trials are beginning to study the safety and tolerability of TRPV4 antagonists in humans [49]. Additionally, there is evidence that TRPV4 interacts with new antidiabetic drugs such as vildagliptin, which could be a route to understand the real potential of these pharmacological agents and their precise indications in the different types of end-organ damage related to diabetes mellitus [50]. The role of TRPV4 channels in the metabolic activity of adipocytes must be considered in future studies that search for and validate alternative strategies to attenuate the impact of metabolic disor- 
ders such as diabetes mellitus. Further characterization of the nature of the molecular mechanisms of these associations would be valuable in determining the therapeutic value of TRPV4 channels as a target in obesity and other metabolic conditions.

\section{CONFLICTS OF INTEREST}

No potential conflict of interest relevant to this article was reported.

\section{ACKNOWLEDGMENTS}

We thank Hospital Universitario San Jorge and Clínica Comfamiliar for allowing us to access its patients and take the samples of adipose tissue.

\section{AUTHOR CONTRIBUTIONS}

Conception or design: J.C.S. Acquisition, analysis, or interpretation of data: J.C.S., A.V.V., A.M.G. Drafting the work or revising: J.C.S., A.V.V., A.M.G. Final approval of the manuscript: J.C.S., A.V.V., A.M.G.

\section{ORCID}

Julio C. Sánchez https://orcid.org/0000-0002-5140-9397

\section{REFERENCES}

1. Cifuentes M, Rojas CV. Antilipolytic effect of calcium-sensing receptor in human adipocytes. Mol Cell Biochem 2008; 319:17-21.

2. Cammisotto PG, Bukowiecki LJ. Role of calcium in the secretion of leptin from white adipocytes. Am J Physiol Regul Integr Comp Physiol 2004;287:R1380-6.

3. Sukumar P, Sedo A, Li J, Wilson LA, O'Regan D, Lippiat JD, et al. Constitutively active TRPC channels of adipocytes confer a mechanism for sensing dietary fatty acids and regulating adiponectin. Circ Res 2012;111:191-200.

4. Goudarzi F, Mohammadalipour A, Khodadadi I, Karimi S, Mostoli R, Bahabadi M, et al. The role of calcium in differentiation of human adipose-derived stem cells to adipocytes. Mol Biotechnol 2018;60:279-89.

5. Schlottmann I, Ehrhart-Bornstein M, Wabitsch M, Bornstein SR, Lamounier-Zepter V. Calcium-dependent release of adipocyte fatty acid binding protein from human adipocytes.
Int J Obes (Lond) 2014;38:1221-7.

6. Amitani M, Asakawa A, Amitani H, Inui A. The role of leptin in the control of insulin-glucose axis. Front Neurosci 2013;7:51.

7. Shan T, Zhang P, Jiang Q, Xiong Y, Wang Y, Kuang S. Adipocyte-specific deletion of $\mathrm{mTOR}$ inhibits adipose tissue development and causes insulin resistance in mice. Diabetologia 2016;59:1995-2004.

8. Pandey GK, Vadivel S, Raghavan S, Mohan V, Balasubramanyam M, Gokulakrishnan K. High molecular weight adiponectin reduces glucolipotoxicity-induced inflammation and improves lipid metabolism and insulin sensitivity via APPL1-AMPK-GLUT4 regulation in 3T3-L1 adipocytes. Atherosclerosis 2019;288:67-75.

9. Zechner R, Madeo F, Kratky D. Cytosolic lipolysis and lipophagy: two sides of the same coin. Nat Rev Mol Cell Biol 2017;18:671-84.

10. Qiao L, Kinney B, Schaack J, Shao J. Adiponectin inhibits lipolysis in mouse adipocytes. Diabetes 2011;60:1519-27.

11. Kim SJ, Tang T, Abbott M, Viscarra JA, Wang Y, Sul HS. AMPK phosphorylates desnutrin/ATGL and hormone-sensitive lipase to regulate lipolysis and fatty acid oxidation within adipose tissue. Mol Cell Biol 2016;36:1961-76.

12. Vincent F, Duncton MA. TRPV4 agonists and antagonists. Curr Top Med Chem 2011;11:2216-26.

13. Hoshi Y, Okabe K, Shibasaki K, Funatsu T, Matsuki N, Ikegaya $\mathrm{Y}$, et al. Ischemic brain injury leads to brain edema via hyperthermia-induced TRPV4 activation. J Neurosci 2018;38:5700-9.

14. Olivan-Viguera A, Garcia-Otin AL, Lozano-Gerona J, Abarca-Lachen E, Garcia-Malinis AJ, Hamilton KL, et al. Pharmacological activation of TRPV4 produces immediate cell damage and induction of apoptosis in human melanoma cells and HaCaT keratinocytes. PLoS One 2018;13:e0190307.

15. Sharma S, Goswami R, Merth M, Cohen J, Lei KY, Zhang DX, et al. TRPV4 ion channel is a novel regulator of dermal myofibroblast differentiation. Am J Physiol Cell Physiol 2017;312:C562-72.

16. Boehmerle W, Huehnchen P, Lee SL, Harms C, Endres M. TRPV4 inhibition prevents paclitaxel-induced neurotoxicity in preclinical models. Exp Neurol 2018;306:64-75.

17. Wu Q, Lu K, Zhao Z, Wang B, Liu H, Zhang S, et al. Blockade of transient receptor potential vanilloid 4 enhances antioxidation after myocardial ischemia/reperfusion. Oxid Med Cell Longev 2019;2019:7283683.

18. White JP, Cibelli M, Urban L, Nilius B, McGeown JG, 
Nagy I. TRPV4: molecular conductor of a diverse orchestra. Physiol Rev 2016;96:911-73.

19. Sanchez JC, Rivera RA, Munoz LV. TRPV4 channels in human white adipocytes: electrophysiological characterization and regulation by insulin. J Cell Physiol 2016;231:954-63.

20. Ye L, Kleiner S, Wu J, Sah R, Gupta RK, Banks AS, et al. TRPV4 is a regulator of adipose oxidative metabolism, inflammation, and energy homeostasis. Cell 2012;151:96-110.

21. Kusudo T, Wang Z, Mizuno A, Suzuki M, Yamashita H. TRPV4 deficiency increases skeletal muscle metabolic capacity and resistance against diet-induced obesity. J Appl Physiol (1985) 2012;112:1223-32.

22. Duan DM, Wu S, Hsu LA, Teng MS, Lin JF, Sun YC, et al. Associations between TRPV4 genotypes and body mass index in Taiwanese subjects. Mol Genet Genomics 2015;290: 1357-65.

23. Palhinha L, Liechocki S, Hottz ED, Pereira JA, de Almeida CJ, Moraes-Vieira PM, et al. Leptin induces proadipogenic and proinflammatory signaling in adipocytes. Front Endocrinol (Lausanne) 2019;10:841.

24. Silha JV, Krsek M, Skrha JV, Sucharda P, Nyomba BL, Murphy LJ. Plasma resistin, adiponectin and leptin levels in lean and obese subjects: correlations with insulin resistance. Eur J Endocrinol 2003;149:331-5.

25. Ramirez-Ponce MP, Mateos JC, Bellido JA. Human adipose cells have voltage-dependent potassium currents. J Membr Biol 2003;196:129-34.

26. Pereira MJ, Thombare K, Sarsenbayeva A, Kamble PG, Almby K, Lundqvist M, et al. Direct effects of glucagon on glucose uptake and lipolysis in human adipocytes. Mol Cell Endocrinol 2020;503:110696.

27. Krycer JR, Quek LE, Francis D, Fazakerley DJ, Elkington $\mathrm{SD}$, Diaz-Vegas A, et al. Lactate production is a prioritized feature of adipocyte metabolism. J Biol Chem 2020;295:8398.

28. Xue B, Greenberg AG, Kraemer FB, Zemel MB. Mechanism of intracellular calcium ([Ca2+] i) inhibition of lipolysis in human adipocytes. FASEB J 2001;15:2527-9.

29. Liedtke W, Choe Y, Marti-Renom MA, Bell AM, Denis CS, Sali A, et al. Vanilloid receptor-related osmotically activated channel (VR-OAC), a candidate vertebrate osmoreceptor. Cell 2000;103:525-35.

30. Ahern GP. Transient receptor potential channels and energy homeostasis. Trends Endocrinol Metab 2013;24:554-60.

31. Yang S, Lu W, Zhao C, Zhai Y, Wei Y, Liu J, et al. Leukemia cells remodel marrow adipocytes via TRPV4-dependent li- polysis. Haematologica 2020;105:2572-83.

32. Snyder PB, Esselstyn JM, Loughney K, Wolda SL, Florio VA. The role of cyclic nucleotide phosphodiesterases in the regulation of adipocyte lipolysis. J Lipid Res 2005;46:494503.

33. Krycer JR, Quek LE, Francis D, Zadoorian A, Weiss FC, Cooke $\mathrm{KC}$, et al. Insulin signaling requires glucose to promote lipid anabolism in adipocytes. J Biol Chem 2020;295: 13250-66.

34. Krycer JR, Yugi K, Hirayama A, Fazakerley DJ, Quek LE, Scalzo R, et al. Dynamic metabolomics reveals that insulin primes the adipocyte for glucose metabolism. Cell Rep 2017;21:3536-47.

35. Cai TQ, Ren N, Jin L, Cheng K, Kash S, Chen R, et al. Role of GPR81 in lactate-mediated reduction of adipose lipolysis. Biochem Biophys Res Commun 2008;377:987-91.

36. Ahmed K, Tunaru S, Tang C, Muller M, Gille A, Sassmann A, et al. An autocrine lactate loop mediates insulin-dependent inhibition of lipolysis through GPR81. Cell Metab 2010;11:311-9.

37. Whitehead JP, Molero JC, Clark S, Martin S, Meneilly G, James DE. The role of $\mathrm{Ca} 2+$ in insulin-stimulated glucose transport in 3T3-L1 cells. J Biol Chem 2001;276:27816-24.

38. Pereira MJ, Palming J, Rizell M, Aureliano M, Carvalho E, Svensson MK, et al. Cyclosporine A and tacrolimus reduce the amount of GLUT4 at the cell surface in human adipocytes: increased endocytosis as a potential mechanism for the diabetogenic effects of immunosuppressive agents. J Clin Endocrinol Metab 2014;99:E1885-94.

39. Fonseca AC, Carvalho E, Eriksson JW, Pereira MJ. Calcineurin is an important factor involved in glucose uptake in human adipocytes. Mol Cell Biochem 2018;445:157-68.

40. Pereira MJ, Palming J, Rizell M, Aureliano M, Carvalho E, Svensson MK, et al. The immunosuppressive agents rapamycin, cyclosporin A and tacrolimus increase lipolysis, inhibit lipid storage and alter expression of genes involved in lipid metabolism in human adipose tissue. Mol Cell Endocrinol 2013;365:260-9.

41. Woody S, Stall R, Ramos J, Patel YM. Regulation of myosin light chain kinase during insulin-stimulated glucose uptake in 3T3-L1 adipocytes. PLoS One 2013;8:e77248.

42. Page AJ, Hatzinikolas G, Vincent AD, Cavuoto P, Wittert GA. The TRPV1 channel regulates glucose metabolism. Am J Physiol Endocrinol Metab 2019;317:E667-76.

43. Tang W, Fan Y. SIRT6 as a potential target for treating insulin resistance. Life Sci 2019;231:116558. 
44. Levy JR, Gyarmati J, Lesko JM, Adler RA, Stevens W. Dual regulation of leptin secretion: intracellular energy and calcium dependence of regulated pathway. Am J Physiol Endocrinol Metab 2000;278:E892-901.

45. Ma X, Qiu S, Luo J, Ma Y, Ngai CY, Shen B, et al. Functional role of vanilloid transient receptor potential 4-canonical transient receptor potential 1 complex in flow-induced Ca2+ influx. Arterioscler Thromb Vasc Biol 2010;30:851-8.

46. Bradley RL, Cheatham B. Regulation of ob gene expression and leptin secretion by insulin and dexamethasone in rat adipocytes. Diabetes 1999;48:272-8.

47. Ozaki KI, Awazu M, Tamiya M, Iwasaki Y, Harada A, Kugisaki S, et al. Targeting the ERK signaling pathway as a potential treatment for insulin resistance and type 2 diabetes. Am J Physiol Endocrinol Metab 2016;310:E643-51.
48. Hu W, Ding Y, Li Q, Shi R, He Y. Transient receptor potential vanilloid 4 channels as therapeutic targets in diabetes and diabetes-related complications. J Diabetes Investig 2020;11:757-69.

49. Goyal N, Skrdla P, Schroyer R, Kumar S, Fernando D, Oughton A, et al. Clinical pharmacokinetics, safety, and tolerability of a novel, first-in-class TRPV4 ion channel inhibitor, GSK2798745, in healthy and heart failure subjects. Am J Cardiovasc Drugs 2019;19:335-42.

50. Gao P, Li L, Wei X, Wang M, Hong Y, Wu H, et al. Activation of transient receptor potential channel vanilloid 4 by DPP-4 (dipeptidyl peptidase-4) inhibitor vildagliptin protects against diabetic endothelial dysfunction. Hypertension 2020;75:150-62. 